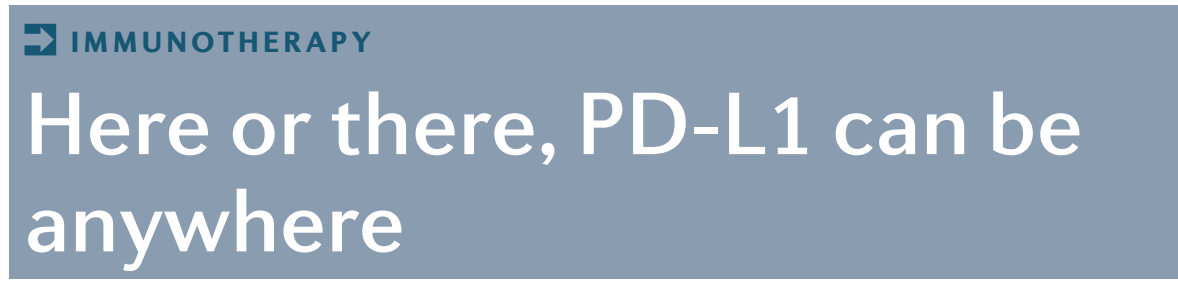

Effective therapies have not been identified for acute-type and lymphoma-type adult T-cell leukaemia/lymphoma (ATLL), the two most aggressive of the four variants of this malignancy. Programmed cell death 1 ligand 1 (PD-L1) expression is associated with a poor prognosis in several solid tumours and haematological malignancies; therefore, Hiroaki Miyoshi and co-workers investigated whether PD-L1 expression could have prognostic value in ATLL. Miyoshi et al. retrospectively analysed 135 biopsy samples from patients with newly diagnosed ATLL and assigned the patients to two main groups according to immunohistochemically confirmed PD-L1 expression: neoplastic PD-L1 (nPD-L1)-positive ( $\geq 50 \%$ PD-L1-stained neoplastic cells; $7.4 \%$ of patients) or nPD-L1-negative; nPD-L1-negative patients were further stratified into microenvironmental PD-L1 (miPD-L1)-positive ( $\geq 10$ stained stromal cells per power field; $58.5 \%$ of patients) and PD-L1-negative (no staining; $34.1 \%$ of patients).
After a median follow-up duration of 10.9 months, the overall survival of patients in the nPD-L1-positive group was inferior to that of the nPD-L1-negative group (7.5 months versus 14.5 months; $P=0.0085)$; among the latter group, superior overall survival was reported for miPD-L1-positive versus PD-L1-negative patients (18.6 months versus 10.2 months; $P=0.0029$ ).

Thus, expression of PD-L1 on cancer cells is associated with poor outcomes in ATLL, probably owing to the immunosuppressive effect of PD-L1. Surprisingly, PD-L1 expression on stromal cells is associated with favourable outcomes through a mechanism that remains unclear. Future studies are needed to elucidate this mechanism, as well as to evaluate the therapeutic potential of anti-PD-1/PD-L1 agents for patients with ATLL.

\section{Diana Romero}

ORIGINAL ARTICLE Miyoshi, H. et al. PD-L1 expression on neoplastic or stromal cell is respectively poor or good prognostic factor for adult T-cell leukemia/lymphoma. Blood http://dx.doi.org/10.1182/blood-2016-02-698936 (2016) 